\title{
TRAITEMENTS ANTICANCEREUX ET SPERMATOGENESE. MOYENS DE PROTECTION
}

\author{
Françoise Grob-Menendez, Dominique Marmor*
}

Unité de Médecine de la Reproduction, Service du Pr Ch. Roux - Hôpital Saint-Antoine - 184, rue du Faubourg Saint-Antoine - 75012 PARIS

*présenté aux 4èmes Journées d'Andrologie de l'Hôpital Saint-Antoine le 15 Juin 1992

\begin{abstract}
ANTINEOPLASIC TREATMENTS AND SPERMATOGENESIS. MEANS OF PROTECTION. A litterature review reports the spermatotoxicity of the different antineoplasic therapies. Recovery of spermatogenesis after completion of most toxic treatments and its delay are individually unforeseen. Pregnancies have occured after treatment with very low sperm counts. These pregnancies ask the question of mutagenicity of antineoplasic treatments. The means of protection seem presently very poor. Key-words : spermatotoxicity - radiotherapy - chemotherapy. Andrologie, 1992, $2:$ : 97-99.
\end{abstract}

\section{INTRODUCTION}

Les progrès de la cancérologie permettent désormais d'espérer la survie, voire la guérison de nombreux patients.

Pour les plus jeunes d'entre eux se pose le problème de leur fertilité ultérieure, qui risque d'être compromise par les diverses modalités thérapeutiques.

Expérimentalement, en effet, tous les traitements anticancéreux ont un effet plus ou moins délétère sur la spermatogénèse. Il persiste toutefois de nombreuses inconnues sur les mécanismes de leur spermatotoxicité, notamment chez l'homme.

En pratique clinique, I'effet des agents anticancéreux s'apprécie par le spermogramme, et plus précisément par la numération spermatique. On observe assez souvent une azoospermie, l'atteinte gonadique étant schématiquement de deux ordres:

- précoce, par atteinte des cellules aux divers stades de la spermatogénèse,

- tardive, par atteinte des cellules souches, cette atteinte étant responsable de la persistance de l'azoospermie plus ou moins longtemps après l'arrêt du traitement (14).

La toxicité gonadique est par ailleurs inégale selon la nature du traitement, sa dose, son rythme et sa durée d'administration.
Avec la plupart des agents anticancéreux, I'azoospermie induite par le traitement est transitoire. D'autres, en revanche sont susceptibles d'entraîner une stérilité définitive (radiations ionisantes, alkylants, procarbazine.. ).

Rappelons enfin que des produits antimitotiques sont utilisés en dehors du domaine oncologique pour leurs propriétés immunosuppressives, avec les mêmes dangers pour la spermatogénèse (tels les alkylants dans le traitement du syndrome néphrotique ).

\section{RISQUES DE LA RADIOTHERAPIE}

Les travaux expérimentaux de ROWLEY \& al. ont bien illustré l'effet d'une irradiation testiculaire unique à dose variable sur la spermatogénèse humaine: apparition d'un oligozoospermie dès $8 \mathrm{cGy}$, d'une azoospermie à partir de $80 \mathrm{cGy}$ (18).

En pratique oncologique, le fractionnement de la dose radiothérapique est de règle, avec un effet notablement plus nocif pour l'épithélium germinal que la délivrance de la même dose en une séance ( apparition d'une azoospermie à partir de 35 cGy )(19). La dose reçue par les gonades au cours de la radiothérapie dépend de nombreux facteurs ( champ d'irradiation, appareillage, dose et débit de dose, anatomie du sujet... ). Dans les conditions matérielles actuelles, on estime qu'une irradiation sus-diaphragmatique est pratiquement dépourvue d'effet sur les gonades. Au cours d'une irradiation sousdiaphragmatique type $\mathrm{Y}$ inversé, les testicules recevraient, selon les auteurs, de 0,4 à $13,6 \%$ de la dose totale délivrée. La seule méthode d'évaluation fiable est la dosimètrie in situ (8), mais elle est en pratique rarement réalisée.

Il est donc difficile d'établir pour un individu donné un pronostic en fonction de la dose reçue, d'autant qu'il semble exister un seuil de sensibilité individuelle variable, aussi bien en ce qui concerne les chances de récupération d'une spermatogénèse que pour son délai et sa qualité.

Les cellules germinales les plus radiosensibles sont les spermatogonies puis, en ordre décroissant, les spermatocytes de 1er ordre, les spermatides. Les spermatozoides, plus matures, sont beaucoup plus résistants. Ainsi s'explique le délai d'apparition de l'azoospermie par rapport au traitement ( souvent de plusieurs semaines ), de même que la survenue possible d'un grossesse au décours immédiat de l'irradiation.

\section{RISQUES DE LA CHIMIOTHERAPIE}

\section{1 -Monochimiothérapie}

La spermatotoxicité des alkylants et de la procarbazine est connue de longue date, tant chez l'animal que chez l'homme, et leur utilisation comporte un risque de stérilité définitive du fait de l'atteinte profonde des spermatogonies souches, seules capables de repeupler un tube séminifère où les cellules germinales ont été détruites (14).

La plupart des autres produits utilisés en monochimiothérapie n'ont qu'un effet modéré ou du moins transitoire sur la spermatogénèse ( méthotrexate, alcaloïdes de la pervenche... ). Les produits plus récents comme le cis-platine sont généralement utilisés en polychimiothérapie, et leurs effets propres sur la spermatogénèse humaine sont mal répertoriés (5).

\section{2 - Polychimiothérapie}

Après protocole ABP (adriamycine + bléomycine + cis-platine) ou VBP (vinblastine + bléomycine + cis-platine) pour cancer non séminomateux du testicule, les chiffres de numération spermatique se normalisent ou retrouvent leur valeur antérieure dans les 18 à 24 mois qui suivent l'arrêt du traitement, voire avant, et il n'y a pas à proprement parler de stérilité séquellaire. Encore faut-il faire la part des troubles de la fertilité pré-existant chez les hommes porteurs d'un cancer du testicule, et celle des troubles liés aux autres modalités thérapeutiques (troubles de l'ejaculation après Iymphadénectomie rétro-péritonéale...)(16).

Le protocole multichimiothérapique AL10, traitement des leucémies aiguës Iymphoblastiques, réparti sur 2 ans et demi - 3 ans utilise du cyclophosphamide en cures brèves et espacées. Les études montrent une dépression marquée de la fonction spermatogénétique au décours des premières phases du traitement, puis un retour à la normale au cours des deuxième et troisième années (12). 
En pratique, le problème le plus souvent rencontré est celui des patients soignés pour maladie de Hodgkin. Traitement de référence depuis les années 70 , le protocole MOPP (chlorméthine + vincristine + procarbazine + prednisone) est à l'heure actuelle le plus spermatotoxique connu: une revue de la littérature en 1986 rapportait plus de $80 \%$ d'azoospermies séquellaires, avec toutefois des délais variables par rapport à la fin du traitement (13).

L'azoospermie apparait habituellement entre le deuxième et le troisième cycle de MOPP. La proportion d'azoospermies durables augmente avec le nombre de cycles, cependant que l'association à une radiothérapie sous-diaphragmatique aggrave par synergie l'atteinte germinale (4).

Depuis 1983, plusieurs études de l'école de Milan, comparant MOPP et ABVD (adriamycine + bléomycine + vinblastine + dacarbazine) rapportent un pourcentage trois fois moindre d'azoospermies après $A B V D$, et surtout une récupération de la spermatogénèse dans les $2-3$ ans suivant le traitement chez la plupart des sujets (1).

\section{STATUT HORMONAL APRES TRAITEMENT}

L'élévation quasi systématique de la FSH plasmatique témoigne de l'atteinte de la fonction spermatogénètique.

Classiquement, la fonction androgénique est respectée, avec une testostéronémie normale ou subnormale. Cependant, la LH plasmatique est assez souvent modérément augmentée; la notion de "compensated Leydig cell failure" a ainsi été développée (22). Enfin, quelques études signalent la survenue d'un hypoandrogénisme après alkylants ou MOPP (6), notamment chez des patients traités en période péri-pubertaire (2I).

\section{REPRISE DE LA FONCTION SPERMATO- GENETIQUE}

Après traitement réputé stérilisant, la récupération spermatique et le délai de celle-ci seraient liés à la dose et à la durée du traitement. On parle ainsi de doses-seuils au delà desquelles la réversibilité de l'atteinte germinale est compromise: 200 cGy en radiothérapie (19), $400 \mathrm{mg}$ au total pour le chlorambucil (17), 119 pour le cyclophosphamide (10), plus de trois cycles pour le MOPP (4). En fait, ces chiffres reposent sur un nombre limité de cas, avec un recul souvent faible, et, en pratique, les chances de récupération d'une spermatogénèse s'avèrent souvent impossibles à prévoir. Le délai de récupération peut dépasser 10 ans.

La reprise de la gamétogénèse s'accompagne généralement d'une diminution voire d'une normalisation des taux de FSH plasmatique. Elle est d'abord histologique puis séminologique.
Elle est souvent partielle avec parfois des chiffres de numération très bas (moins de 5 millions de spermatozoïdes par ml). La mobilité ou la morphologie des spermatozoides ne semblent pas affectés au long cours.

Il est fondamental de rappeler qu'il est régulièrement fait mention dans la littérature de conceptions obtenues après traitement anticancéreux avec des numérations spermatiques très faibles, tant chez l'animal que chez l'homme $(3,9)$. Ainsi, à partir du moment où des spermatozoïdes mobiles sont présents dans l'éjaculat, les chiffres du spermogramme n'ont pas de valeur pronostique sur la fertilité.

\section{MUTAGENESE}

La survenue de grossesses pose le problème du risque mutagène lié aux traitements anticancéreux. Expérimentalement, tous les agents antinéoplasiques sont mutagènes, augmentant la fréquence des mutations spontanées, chromosomiques ou géniques, dominantes ou récessives.

Le risque mutagène est plus important au décours immédiat du traitement car il affecte des cellules germinales non détruites par celui-ci, c'est-à-dire les plus matures.

Ultérieurement, le risque, porté par les cellules souches est définitivement acquis; il est cumulatif et transmissible à la descendance.

Différent de l'effet tératogène, l'effet mutagène est très difficile à prouver ou à nier dans l'espèce humaine car un long délai d'observation est nécessaire (plusieurs générations pour une mutation récessive); surtout, les traitements mutagènes augmentent la fréquence des mutations spontanées, mais n'en font pas apparaître de nouvelles.

En tout état de cause, ce risque parait faible (20), ne justifiant absolument pas le recours systématique à l'interruption de grossesse.

Il convient de rassurer les couples et de proposer une surveillance de la grossesse par des échographies de haute qualité, voire une amniocentèse précoce.

\section{MOYENS DE PROTECTION}

lls s'avèrent actuellement limités.

L'hypothèse principale qui sous-tend le souci de protéger l'épithélium germinal est de le rendre moins vulnérable en y stoppant les divisions cellulaires. Dans ce but, divers produits hormonaux ont particulièrement été étudiés ces dernières années. En 1981, GLODE 1 al. rapportaient l'effet protecteur d'un agoniste de la LH-RH chez des souris recevant du cyclophosphamide (7). Depuis cette date, plusieurs équipes ont publiés des résultats, souvent contradictoires, chez différentes espèces animales (15).
Chez l'homme, aucun protocole hormonal n'a permis à ce jour d'atténuer la spermatotoxicité des traitements anticancéreux. Ceci remet en cause l'hypothèse d'une moindre sensibilité des gonades "quiescentes".

En pratique, à l'heure actuelle, force est de limiter les dégâts:

- par l'utilisation systématiques de caches testiculaires plombés lors des séances de radiothérapie. La dose reçue par les gonades peut ainsi être diminuée de moitié (11);

- par l'utilisation à bon escient de la chimiothérapie et le choix, dans la mesure du possible, des produits et/ou des protocoles les moins spermatotoxiques;

- enfin et surtout, en proposant systématiquement la cryoconservation préalable du sperme lorsqu'elle est possible.

\section{CONCLUSION}

Certains traitements anticancéreux comportent un risque de stérilité séquellaire. Il est donc nécessaire de s'en préoccuper.

Cependant, il n'existe pas de stérilité définitive systématique, et la récupération de la spermatogénèse après traitement anticancéreux et le délai de celle-ci peuvent s'avérer imprévisibles au plan individuel.

Des grossesses sont possibles après traitement avec des numérations spermatiques très basses: celles-ci nous parait donc peu fiable pour évaluer la fertilité d'un homme ayant subi ce type de traitement. Il convient aussi de se souvenir que la fécondité est affaire de couple, ce qui souligne dans ce contexte toute l'importance de la fertilité de la conjointe.

Enfin, le problème de la mutagénèse liée à l'utilisation des thérapeutiques anticancéreuses reste posé.

\section{REFERENCES}

1 - Bonnadonna G., Santoro A., Viviani S., Lombardi C., Ragni G. Gonadal damage in Hodgkin's disease from cancer chemotherapeutic regimens. Arch.Toxicol. , 1984,sup7:140

2- Chapman R.M., Sutcliffe S.B., Rees L.H., Edwards C.R.W., Malpas J.S. Cyclical combination chemotherapy and gonadal function. Lancet, 1979,i:285

3- Chapman R.M., Sutcliffe S.B., Crosby W.H Reproductive potential after treatment for Hodgkin's disease. N.Eng.J.Med. , 1981,305:890

4- Da Cuhna M.F., Meistrich M.L., Fuller L.M., Cundiff J.H., Hagemeister F.B., Velasquez W.S., Mc Laughlin P., Riggs S.A., Cabanillas F.F., Salvador P.G. Recovery of spermatogenesis after treatment for Hodgkin's disease: limiting dose of Mopp chemotherapy. J.Clin.Oncol , 1984,2:571. 
5 - Damewwod M.D., Grochow L.B. Prospects for fertility after chemotherapy or radiation for neoplastic disease. Fertil.Steril. , 1986,4:443

6 - Friedmann N.M., Plymate S.R. Leydig cell dysfunction and gynecomastia in adult males treated with alkylating agents. Clin.Endoc. , 1980, 12:553

7 - Glode L.M., Robinson J., Gould S.F. Protection from cyclophosphamide induced testicular damage with an analog of gonadotrophin releasing hormone. Lancet, 1981,i:1132

8 - Hahn E.W., Feingold S.M., Niscel L. Aspermia and recovery of spermatogenesis in cancer patients following incidental gonadal irradiation during treatment: a progress report. Radiology 1976,119:223

9 - Hahn E.W., Feingold S.M., Simpson L., Batata M. Recovery from aspermia induced by low dose radiation in seminoma patients. Cancer, 1982,50:337

10 - Hsu E.W., Folami A.O., Bain J., Rance C.P. Gonadal function in males treated with cyclophosphamide for neoplastic syndrome. Fertil.Steril. 1979,31:173

11 - Jackson H., Hass C.A., Sooby D., Marschke C.H. The gonadal exposure of boys and young men treated with inverted $Y$ fields. Radiology 1970,96:181

12 - Kreuser E.D., Hetzel W.D., Heit W., Hoelzer D., Eurrle E., Xiros N., Heimpel H. Reproductive and endocrine function gonadal functions in adults following multidrug chemotherapy for acute Iymphoblastic or undifferentiated leukemia. J.Clin.Oncol. , 1988,6:588

13- Marmor D. Influence de la maladie de Hodgkin sur les fonctions reproductrices. In: Progrès en Hématologie. Hoerni B., Simon G, Gisselbrech C. ed. Doin Paris, 1985: 165

14 - Meistrich M.L. Relationship between spermatogonial stem cell survival and testis function after cytotoxic therapy. Br.J.Cancer, 1986,53:89

15 - Morris 1.D., Shalet S.M. Protection of gonadal function from cytotoxic chemotherapy and irradiation. Baill.Clin.Endoc.Metab. , 1990,4:97

16 - Pontonnier S., Navratil H. Stérilité masculine et cancer du testicule. J.Urol. , 1984,90:308

17 - Richter P., Calamera J.C., Morgenfield M.C., Kierszenbaum A.L., Lavieri J.C., Mancini R.E. Effects of chlorambucil on spermatogenesis in the human with malignant Iymphoma. Cancer, 1970,25:1026

18 - Rowley M.J., Leach D.R., Warner G.A., Heller C.G. Effects of graded doses of ionizing radiations on the human testis. Radiat.Res., 1974,59:665

19 - Sandeman T.F. The effects of X irradiation on the human fertility. Brit.J.Radiol. , 1966,39:901

20 - Schweiguth O. L'avenir des enfants guéris d'un cancer. Arch.Fr.Pediatr. , 1985,42:3
21 - Sherins R.J., Olweny C.L.M., Ziegler J.L Gynaecomastia and gonadal dysfunction in adolescent boys treated with combination chem therapy for Hodgkin's disease. N.Engl.J.Med. , 1978,299:12

22 - Withehead E., Shalet S.M., B1ckledge G., Tood 1., Crowther D., Beardwell C.G. The effects of Hodgkin's disease and combination chemotherapy on gonadal function in the adult male. Cancer 1982,49:418

RESUME : Une revue de la littérature fait le point de la spermatotoxicité des différentes thérapeutiques anticancéreuses. Après les traitements réputés stérilisants, la récupération de la fonction gamétogénétique et son délai s'avèrent imprévisibles au plan individuel. Des grossesses ont été obtenues après traitement anticancéreux avec des chiffres de numération spermatique très bas. Celles-ci posent le problème de la mutagénicité des thérapeutiques anticancéreuses. Les moyens de protection s'avèrent actuellement limités. Mots clés : spermatotoxicité radiothérapie - chimiothérapie. Andrologie, 1992, 2 : 97.99. 